\title{
Mediastinite pós-cirúrgica em um Hospital Cardiológico de Recife: contribuições para a assistência de enfermagem
}

\author{
POST-OPERATIVE MEDIASTINITIS IN A HEART HOSPITAL OF RECIFE: \\ CONTRIBUTIONS FOR NURSING CARE
}

\section{MEDIASTINITIS POSTOPERATORIA EN UN HOSPITAL CARDIOLÓGICO DE RECIFE: CONTRIBUCIONES PARA LAATENCIÓN DE ENFERMERÍA}

\section{Marina Gabriella Pereira de Andrada Magalhães' ${ }^{1}$, Ludmila Medeiros Outtes Alves², Lidianne Fábia de Moraes Alcantara ${ }^{3}$, Simone Maria Muniz da Silva Bezerra ${ }^{4}$}

\section{RESUMO}

O objetivo deste estudo foi determinar a prevalência de mediastinite pós-cirúrgica com o intuito de contribuir para a assistência de enfermagem. Para tanto, foi realizada a análise de 896 prontuários de pacientes submetidos à operação cardíaca com esternotomia no Pronto Socorro Cardiológico de Recife-PE,no período de junho de 2007 a junho 2009. As variáveis consideradas foram: sexo, idade, tipo de operação, antecedentes pessoais, tempo de internamento hospitalar, uso de antibióticos e cultura de ferida operatória. Observou-se alta letalidade por mediastinite (33,3\%). Vários fatores de risco foram identificados no estudo, tais como: hipertensão arterial sistêmica (80,9\%); tabagismo (61,9\%); diabetes mellitus $(42,8 \%)$ e obesidade $(33,3 \%)$, sendo a maioria $(76,2 \%)$ em pacientes submetidos à cirurgia de revascularização do miocárdio. Conclui-se que a mediastinite é uma infecção grave que necessita de supervisão contínua da enfermagem e medidas preventivas para o diagnóstico precoce e a diminuição da mortalidade.

\section{DESCRITORES}

Mediastinite

Osteomielite

Cirurgia torácica

Enfermagem perioperatória

\begin{abstract}
The objective of this study was to determine the prevalence of post-operative mediastinitis with the purpose to contribute to nursing care knowledge. To do this, an analysis was performed on 896 medical records of patients who underwent heart surgery involving sternotomy at the Cardiology Emergency Room of Recife-PE, in the period between June 2007 and June 2009. The following variables were considered: gender, age, type of surgery, personal history, length of stay, use of antibiotics, and culture of the surgical wound. A high death rate from mediastinitis was observed (33.3\%). Several risk factors were identified, including: systemic arterial hypertension (80.9\%); smoking (61.9\%); diabetes mellitus (42.8\%); and obesity (33.3\%), most of which $(76.2 \%)$ were identified in patients who underwent surgery for myocardial revascularization. It is concluded that mediastinitis is a serious infection that needs continuous nursing supervision and preventive measures to assure an early diagnosis and, thus, reduce mortality.
\end{abstract}

DESCRIPTORS
Mediastinitis
Osteomyelitis
Thoracic surgery
Perioperative nursing

\begin{abstract}
RESUMEN
Este estudio objetivó determinar la prevalencia de mediastinitis postoperatoria con la intención de contribuir a la atención de enfermería. Fueron analizadas 896 historias clínicas de pacientes sometidos a operación cardíaca con esternotomía en Urgencia Cardiológica de Pernambuco, en el período de junio 2007 a junio 2009. Las variables consideradas fueron: sexo, edad, tipo de operación, antecedentes personales, tiempo de internación hospitalaria, uso de antibióticos y cultivo de la herida operatoria. Se observó alta mortalidad por mediastinitis (33,3\%). Fueron identificados en el estudio algunos de los factores de riesgo, tales como: hipertensión arterial sistémica (80,9\%), tabaquismo (61,9\%), diabetes mellitus $(42,8 \%)$ y obesidad $(33,3 \%)$, ocurriendo la mayoría $(76,2 \%)$ en pacientes sometidos a cirugía de revascularización del miocardio. La mediastinitis es una infección grave que necesita de supervisión continua de enfermería y medidas preventivas para el diagnóstico precoz y disminución de la mortalidad.
\end{abstract}

\section{DESCRIPTORES \\ Mediastinitis \\ Osteomielitis \\ Cirugía torácica \\ Enfermería periperatoria}

\footnotetext{
'Enfermeira. Graduada em Enfermagem pela Faculdade de Enfermagem Nossa Senhora das Graças da Universidade de Pernambuco. Recife, PE, Brasil. gabi.magalhaes@hotmail.com ${ }^{2}$ Enfermeira. Graduada em Enfermagem pela Faculdade de Enfermagem Nossa Senhora das Graças da Universidade de Pernambuco. Recife, PE, Brasil. pcmila@hotmail.com ${ }^{3}$ Enfermeira. Graduada em Enfermagem pela Faculdade de Enfermagem Nossa Senhora das Graças da Universidade de Pernambuco. Recife, PE, Brasil. lidifabi@hotmail.com ${ }^{4}$ Doutora em Ciências pela Universidade de São Paulo. Professora Adjunta do Departamento Médico Cirúrgica da Faculdade de Enfermagem Nossa Senhora das Graças da Universidade de Pernambuco. Recife, PE, Brasil. simonemuniz@upe.fensg.br
} 


\section{INTRODUÇÃO}

As infecções hospitalares de maior prevalência caracterizam-se pela instalação e desenvolvimento de microorganismos na incisão operatória( ${ }^{(1)}$. A mediastinite pós-operatória possui patogenia complexa e multifatorial, sendo definida como uma infecção e/ou inflamação do tecido conjuntivo do mediastino associada à osteomielite do esterno, com ou sem sua instabilidade, podendo atingir ainda o espaço retroesternal ${ }^{(2,3-4)}$. Pode ser causada por esternotomia mediana ${ }^{(5-6)}$, ruptura esofágica ${ }^{(7-8)}$, infecções cervicais profundas e de forma rara, por empiema pleural, osteomielite vertebral ou costal, abscessos retroperitoneais e subfrênicos ${ }^{(4-5,9)}$.

Os fatores associados ao desenvolvimento da infecção na cirurgia cardíaca, geralmente estão relacionados ao paciente (colonização da pele, obesidade, Diabetes Mellitus, idade, tempo do internamento pré-operatório, infecções pré-existentes, índice de gravidade da doença, etc.) e/ou aos procedimentos cirúrgicos (isquemia ${ }^{(10)}$, aproximação inadequada das bordas da ferida, presença de tecido necrótico ou corpo estranho, tempo de cirurgia, etc. $)^{(1,11)}$. O tipo e o grau da contaminação da ferida operatória (FO) dependem também da resposta sistêmica fisiológica, liberação de mediadores inflamatórios, suprimento sanguíneo local, nutrição, estado imunológico do paciente ${ }^{(11)}$, entre outros.

Fatores como obesidade associada ao diabetes, principalmente em pacientes que fazem uso de insulinoterapia, reoperação com tempo maior de perfusão e politransfusão sanguínea têm sido fatores de risco elevados ${ }^{(8,11-12)}$, que contribuem para o aparecimento da mediastinite ${ }^{(3,11)}$, sendo a utilização de duas pontes mamárias um fator muito discutido, uma vez que, predispõe a desvascularização do esterno, tornando-o um ambiente propício para o aparecimento de infecção $0^{(3,8,11)}$.

Dentre os agentes infecciosos encontrados nas culturas de tecido mediastinal inflamado, destacam-se o Staphylococcus aureus, o Staphylococcus epidermidis, as Pseudomonas sp e a Escherichia coli, sendo esta última associada a um alto índice de mortalidade ${ }^{(9,13)}$. Porém, ainda são relatadas infecções mistas ${ }^{(8)}$. Em alguns casos, a cultura do exsudato mediastinal pode ser negativa, o que na maioria das vezes se deve ao uso prévio de antibióticos ${ }^{(5)}$.

A incidência de mediastinite pós-operatória varia de acordo com a rotina da instituição, uso de antimicrobianos profiláticos, padronização de técnicas assépticas, etc, sendo estimada de 0,4 e 5,0\%, com média de 2,0\%, conforme o Guidelines do American College of Cardiology e da American Heart Association (ACC/AHA) ${ }^{(14)}$. A taxa de mortalidade parece estar relacionada com o atraso no diagnóstico e início do tratamento, elevando essa freqüência entre 10,0 e $47,0 \%{ }^{(3)}$.

Os sinais e sintomas apresentados pelos pacientes com mediastinite variam desde febre persistente após o quarto dia de pós-operatório, toxemia, leucocitose, bacteremia, secreção purulenta e deiscência da ferida cirúrgica (ocorrendo em $70 \%$ a $90 \%$ dos casos), normalmente associada à dor torácica e instabilidade esternal|(1,8,12).

O diagnóstico é geralmente tardio, necessitando da confirmação através de pelo menos dois dos seguintes achados: identificação de microorganismos no fluido coIhido do espaço mediastinal, cultura dos tecidos mediastinais, evidência radiológica de infecção e deiscência da ferida operatória esternal|(15). Entretanto, o diagnóstico definitivo é realizado através de punção esternal ou da própria exploração da ferida cirúrgica ${ }^{(3,12)}$.

O seu tratamento varia desde a terapia com antimicrobianos até a completa esternectomia e procedimentos plásticos reconstrutivos da parede torácica ${ }^{(1,3,12)}$. Nos pacientes em que ocorre infecção da ferida mediastinal com acometimento apenas subcutâneo, o tratamento consiste na abertura inicial da lesão e análise criteriosa do processo infeccioso e sua extensão, uso direcionado de antimicrobianos seguindo concomitantemente os princípios de tratamento da ferida aberta com curativos $^{(1)}$.

Pelo fato das infecções hospitalares estarem ligadas aos fatores relacionados ao paciente e ao procedimento, a equipe multiprofissional desempenha papel fundamental no que concerne a prevenção das infecções no âmbito hospitalar, o que exige da equipe de saúde e das instituições, medidas preventivas, treinamento em serviços, mudanças de atitudes e educação continuada como ponto fundamental do processo assistencial. Ademais, os custos oriundos da internação e tratamento de um paciente acometido pela mediastinite mostram-se três vezes mais dispendiosos do que os de um paciente sem infecção ${ }^{(1,12)}$.

Com o avanço das tecnologias de assistência hospitalar, surgiram as primeiras Comissões de Controle de Infecção Hospitalar (CCIH). Em 1997 o Ministério da Saúde (MS) aprovou a lei 9431/97, que tornava obrigatória a presença da Comissão de Controle de Infecção Hospitalar e Programas de Controle de infecção hospitalar em todos os hospitais, independente da complexidade do atendimento prestado. No ano seguinte, a portaria 2616/98, do Ministério da Saúde torna obrigatória a presença do enfermeiro na equipe de profissionais que compõem a Comissão de Controle de Infecção Hospitalar ${ }^{(16)}$.

A responsabilidade do cuidar exige que as decisões sobre as intervenções sejam pautadas na avaliação do 
estado de saúde do doente, na situação clínica e na natureza das informações que se quer obter. As habilidades cognitivas e perceptivas da enfermagem são fatores que influenciam a assistência e a identificação precoce dos sinais e sintomas ${ }^{(2,17)}$.

Este estudo torna-se de grande importância por colaborar com dados que podem ser utilizados na identificação de grupos de risco para desenvolvimento da morbidade e na elaboração de métodos preventivos na infecção do mediastino e na criação de um plano terapêutico pela equipe de enfermagem, tendo em vista a escassez de estudos a respeito dessa patologia no Brasil, principalmente na região Nordeste.

\section{OBJETIVO}

Investigar a prevalência de mediastinite pós-cirúrgica nos pacientes internados no Pronto Socorro Cardiológico de Pernambuco (PROCAPE) com o intuito de contribuir com a assistência de enfermagem.

\section{MÉTODO}

Estudo retrospectivo, descritivo, transversal com abordagem quantitativa, realizado no período de junho de 2007 a junho de 2009, no Pronto Socorro Cardiológico da Universidade de Pernambuco, onde foram analisados todos os prontuários de pacientes com diagnóstico de mediastinite. As informações foram coletadas através de dados fornecidos pela Comissão de Controle de Infecção Hospitalar do referido hospital. Este setor estabelece normas e rotinas que visam desenvolver, orientar e supervisionar as ações a serem executadas pelos profissionais que atuam na área da saúde e outros profissionais que desenvolvem atividades afins, no sentido de minimizar o desenvolvimento e/ou ocorrência de infecção hospitalar.

As variáveis utilizadas no presente estudo foram: gênero, idade, diabetes mellitus (DM), doença pulmonar obstrutiva crônica (DPOC), obesidade (IMC $\geq 30 \mathrm{~kg} / \mathrm{m}^{2}$ ), tabagismo, cirurgia cardíaca prévia, tempo de circulação extracorpórea (> 120 minutos), de cirurgia e internamento em Unidades de Terapia Intensiva (UTI), politransfusão sanguínea no pós-operatório e uso de ponte mamária.

Foram limitações do estudo a identificação de cor, ocupação e nível de escolaridade nas amostras devido a falta de dados e preenchimento incompleto de prontuários, sendo essas variáveis excluídas da análise.

As variáveis estudadas foram armazenadas em programa de computador EPI-INFO versão 3.5.1/2008 - Atlanta/ EUA, a partir do qual foram realizados cálculos estatísticos com análise e interpretação.

Foi utilizado um instrumento de coleta de dados composto de perguntas objetivas, tipo check list, preenchido pelas pesquisadoras através de consultas nos prontuários catalogados no arquivo médico do hospital. Foram utilizados dados de identificação, história da doença atual e pregressa, diagnóstico da doença atual, tipo de cirurgia, dados pré, trans e pós-operatórios, recuperação, tempo de internação e alta hospitalar. A aplicação do instrumento ocorreu entre os meses de janeiro a abril de 2010.

O projeto de pesquisa foi aprovado pelo Comitê de Ética em Pesquisa (CEP) do Hospital Universitário Oswaldo Cruz (HUOC) sob o protocolo: 5066.0.000.106-09. A mediastinite foi definida com base no diagnóstico presente na evolução médica.

\section{RESULTADOS}

De acordo com os relatórios de controle de infecção hospitalar do Pronto Socorro Cardiológico da Universidade de Pernambuco no período de Junho de 2007 à Junho de 2009 foram realizadas 896 cirurgias cardíacas. De acordo com os dados obtidos através da Comissão de Controle de Infecção Hospitalar, foram analisados 35 prontuários de pacientes com diagnóstico de mediastinite. Destes, quatro prontuários não foram encontrados no arquivo médico, sete não tiveram diagnóstico confirmando infecção da cavidade torácica e três pacientes realizaram cirurgia em outro hospital, os quais foram excluídos da pesquisa. Portanto, fizeram parte do estudo 21 prontuários de pacientes com diagnóstico médico confirmado de mediastinite pós-operatória internados no Pronto Socorro Cardiológico da Universidade de Pernambuco no período de junho de 2007 a junho de 2009.

As enfermeiras da Comissão de Controle de Infecção Hospitalar, neste hospital, responsáveis pela primeira avaliação da ferida cirúrgica com sinais de infecção, orientam previamente os pacientes, que após a alta deverão comparecer ao hospital para acompanhamento quanto à dieta, limpeza e troca de curativos, a fim de dar continuidade ao tratamento e evitar reinternações desnecessárias. A partir dessa primeira avaliação, são iniciados os protocolos utilizados pelas enfermeiras no controle de infecção, que consiste na solicitação do parecer do médico-cirurgião quanto à inspeção da cavidade torácica, presença de exsudatos, osteomielite, investigação de sinais e sintomas de infecção, exames de imagem, laboratoriais e avaliação clínica do estado geral do paciente para definição do caso. Após confirmação, dá-se início ao tratamento através de uma anamnese e exame físico, observação criteriosa da área infectada, definição do tipo de curativo e do uso de antimicrobianos, se necessário.

Comparativamente com as demais infecções, a mediastinite apresenta posição de destaque, neste estudo. As infecções pós-operatórias obtiveram um total de $96 \mathrm{ca}$ sos (10,7\%). Destes, 21 (2,3\%) foram de mediastinite após operação cardíaca com esternotomia, com 7 óbitos (33\%) (Figura1). 


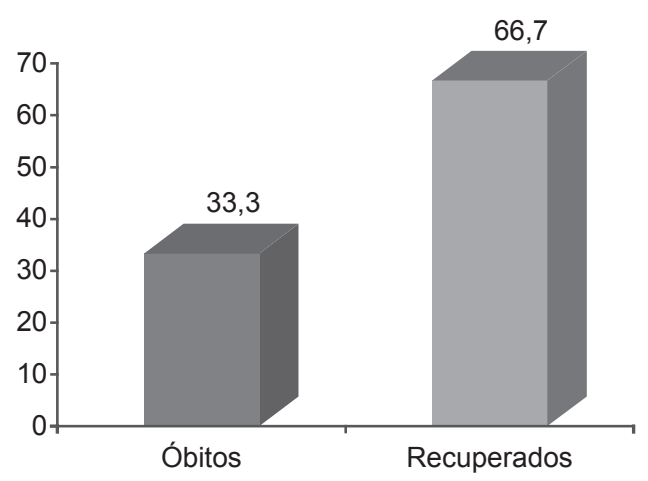

Figura 1 - Frequência de óbitos ocorridos e pacientes recuperados no Pronto Socorro Cardiológico da Universidade de Pernambuco Recife, PE, 2007/2009

A operação de revascularização do miocárdio (CRVM) esteve relacionada com a ocorrência de $76,2 \%$ dos casos de mediastinite (16 pacientes), enquanto que o restante ocorreu após operação de correção de dissecção (3 casos) e troca valvar (2 pacientes) (Figura 2). A correlação entre operações de revascularização de miocárdio e o desenvolvimento de mediastinite está bem documentada na literatura, onde se aponta mais de $80 \%$ de casos da infecção como complicação associada a esta operação( ${ }^{(8,19)}$.

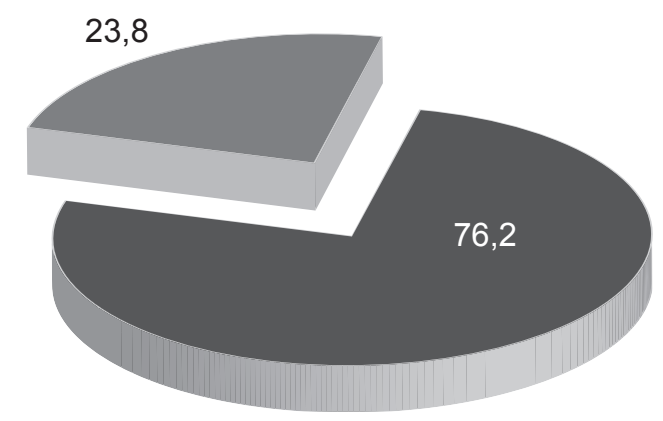

Figura 2 - Prevalência de cirurgias de revascularização do miocárdio realizadas no Pronto Socorro Cardiológico da Universidade de Pernambuco - Recife, PE - 2007/2009

Quanto ao sexo, 52,4\% dos pacientes eram do sexo masculino, enquanto que $10(47,6 \%)$ eram do sexo feminino. A idade média correspondeu a $60,04 \pm 15,60$, variando entre 1 e 77 anos. Quanto à procedência, 66,6\% foram da capital e 33,4\% do interior (Tabela 1). O período com o maior número de casos apresentados foi no ano de 2007, correspondendo a um total de $38,1 \%$, enquanto que nos períodos subseqüentes foram observadas reduções significativas de números de casos, $33,4 \%$ e $28,5 \%$.

Houve predominância de fatores de co-morbidade: a hipertensão arterial sistêmica (HAS) esteve presente em 80,9\% dos casos; o tabagismo foi evidenciado em $61,9 \%$; o diabetes mellitus em $42,8 \%$; a obesidade apresentou $33,3 \%$ dos casos. Os demais não apresentavam doenças importantes associadas àquela que motivou o ato cirúrgico e que correspondessem fator de risco para o desenvolvimento de mediastinite.
Tabela 1 - Perfil epidemiológico dos pacientes que desenvolveram mediastinite no Pronto Socorro Cardiológico da Universidade de Pernambuco - Recife, PE - 2007/2009

\begin{tabular}{lcc}
\hline Variáveis (n= 21) & \multicolumn{2}{c}{ Total } \\
\hline Sexo & N & $\mathbf{\%}$ \\
Masculino & 11 & 52,4 \\
Feminino & 10 & 47,6 \\
\hline Idade (anos) & & \\
$0-40$ & 02 & $\mathbf{9 , 5}$ \\
$41-60$ & 08 & $\mathbf{3 8 , 1}$ \\
$61-80$ & 11 & $\mathbf{5 2 , 4}$ \\
\hline Procedência & & \\
Capital & 14 & $\mathbf{6 6 , 7}$ \\
Interior & 07 & $\mathbf{3 3 , 3}$ \\
\hline Antecedentes Pessoais & \\
Hipertensão Arterial Sistêmica & & \\
Etilismo & 17 & $\mathbf{8 0 , 9}$ \\
Alergia & 02 & $\mathbf{9 , 5}$ \\
Diabetes Mellitus & 05 & $\mathbf{2 3 , 8}$ \\
Tabagismo & 09 & $\mathbf{4 2 , 8}$ \\
Doença Pulmonar Obstrutiva Crônica & 13 & $\mathbf{6 1 , 9}$ \\
Obesidade & 01 & $\mathbf{4 , 8}$ \\
Cirurgia Cardíaca Prévia & 07 & $\mathbf{3 3 , 3}$ \\
Outros & 05 & $\mathbf{2 3 , 8}$ \\
\hline
\end{tabular}

*Nota: O mesmo prontuário apresentava mais de um dos antecedentes pessoais.

Quanto à preparação da pele do paciente no período pré-operatório, todos os pacientes tiveram a higiene corporal realizada, sugerindo a diminuição da colonização da pele. Não foram identificadas lesões tissulares, apesar de $20 \%$ dos pacientes já estarem acamados antes mesmo da cirurgia.

O uso de antibioticoterapia profilática é indicada em cirurgias de grande porte ou em pacientes com risco de desenvolver infecções e imunodeprimidos. Neste caso, observa-se que a profilaxia pré e intra-operatória com o uso de antibióticos, apesar de conhecida na prática, não foi evidenciada nos prontuários analisados, existindo poucas informações escritas a respeito.

Nas cirurgias cardíacas com duração entre 4 e 5 horas foram encontradas, pela pesquisa, taxas mais elevadas associadas ao desenvolvimento de infecção no mediastino ( $35,3 \%$ e $29,4 \%$ respectivamente). Dados da literatura apontam que o tempo intra-operatório desta cirurgia é de aproximadamente cinco horas ${ }^{(20)}$.

A mediastinite esteve associada com o uso de marcapasso externo em $23,8 \%$ dos casos. O mesmo é necessário apenas para pacientes com risco de distúrbios de condução cardíaca, tendo apresentado baixa porcentagem nos casos analisados. Este, porém, é um importante fator de risco, já que cria uma ligação entre o meio externo e o interior da cavidade torácica, através do fio externo, podendo constituir uma via de infecção.

O tempo de internação total dos pacientes variou entre 16 e 158 dias, ficando a maior porcentagem no inter- 
valo de 41 a 100 dias (50,0\%). Contudo, foi observado que grande parte dos pacientes $(50,0 \%)$ esteve internada por mais de 20 dias antes de realizar a primeira cirurgia, contribuindo para o elevado tempo de internação.

Outro fator que chama a atenção é o elevado tempo de recuperação pós-operatória encontrado (21 a 120 dias), estando bastante elevado em comparação com o índice médio de tempo de recuperação em cirurgias de grande porte encontrado em estudos ( 7 a 14 dias na literatura, sendo a média de 11 dias) ${ }^{(21)}$. Isso pode ser relacionado com o tempo decorrido entre a cirurgia e o surgimento dos primeiros sinais e sintomas (variando entre 2 dias e 4

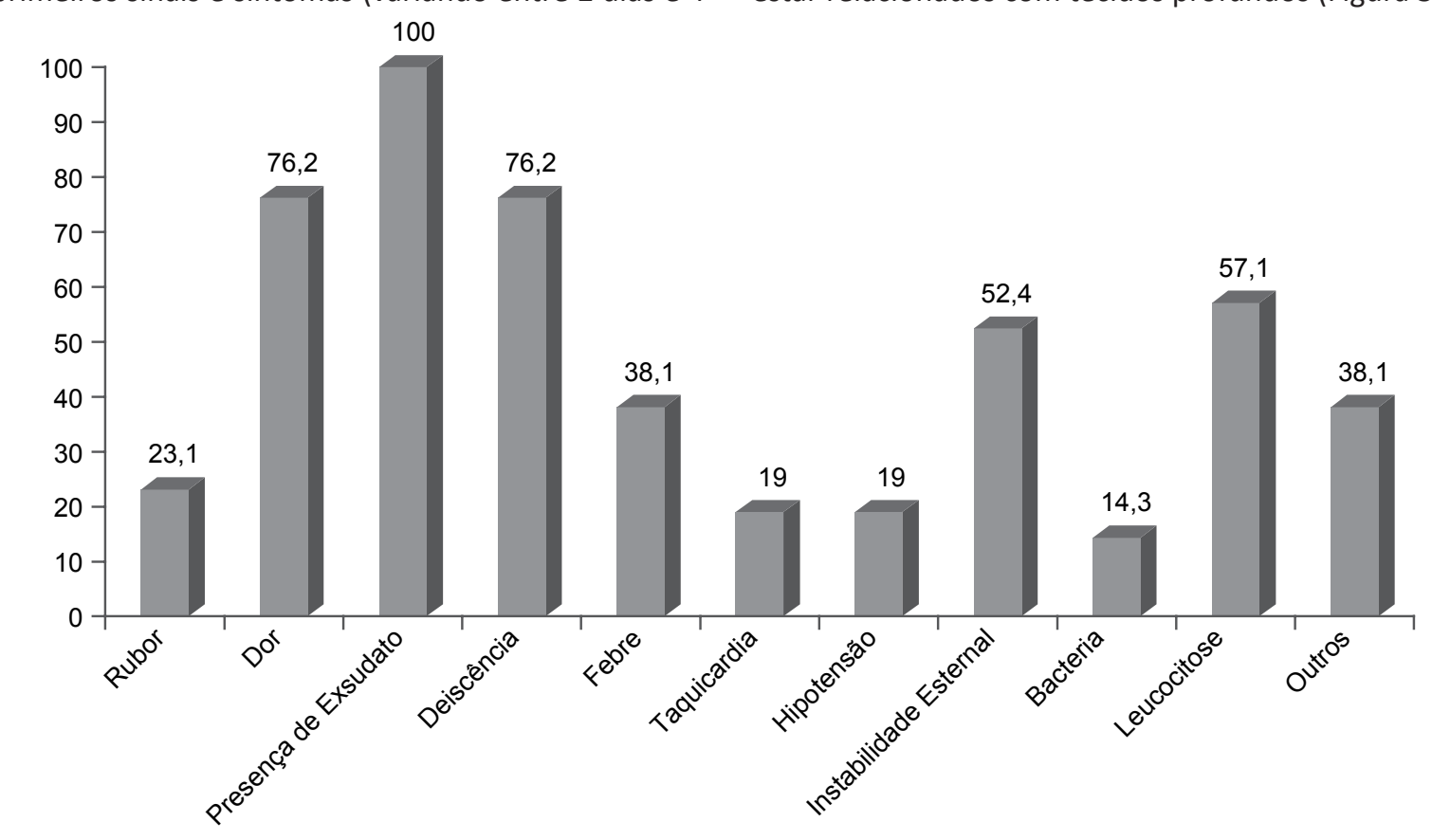

*Nota: O mesmo prontuário apresentava mais de um dos sinais e sintomas semanas), a demora para o diagnóstico e a complexidade da doença, que exige tratamentos longos.

Os sinais e sintomas mais encontrados na amostra do estudo foram a presença de exsudato, com $100,0 \%$, e deiscência da ferida operatória e dor, com 76,2\%, cada. Os pacientes acometidos por mediastinite apresentaram sinais e sintomas entre 3 e 19 dias. Os sinais sistêmicos, como febre, leucocitose e taquicardia, são fortes indicativos de infecção cavitária, ao passo que sinais flogísticos locais, como dor, rubor e presença de exsudato, apenas indicam inflamação da ferida operatória, podendo ou não estar relacionados com tecidos profundos (Figura 3).

Figura 3 - Sinais e sintomas apresentados em pacientes que desenvolveram mediastinite no Pronto Socorro Cardiológico da Universidade de Pernambuco - Recife, PE - 2007/2009

A distância entre a data de realização da cirurgia e o aparecimento dos primeiros sinais e sintomas pode ser tão elevada, que o paciente recebe alta antes que seja diagnosticada a mediastinite, sendo refletido no alto índice encontrado de reinternação $(23,8 \%)$.

Dentre os métodos utilizados para confirmação diagnóstica da doença, o mais comum foi o clínico $(43,0 \%)$, seguido da cultura $(35,0 \%)$, sendo esta última realizada através do tecido da lesão ou do exsudato. Normalmente o diagnóstico é confirmado com a junção de dois ou mais métodos.

Assim que ocorre a suspeição do quadro de mediastinite, inicia-se um esquema de antibioticoterapia, que pode ser modificado após a cultura, no caso de bacilos resistentes. $O$ resultado desta confirma a presença de infecção, bem como seu agente causador, que pode ser mais de uma bactéria, sendo conhecida como infecção mista. Os germes mais frequentemente isolados foram $S$. aureus $(25,0 \%)$ e $K$. pneumoniae (21\%) (Figura 4).

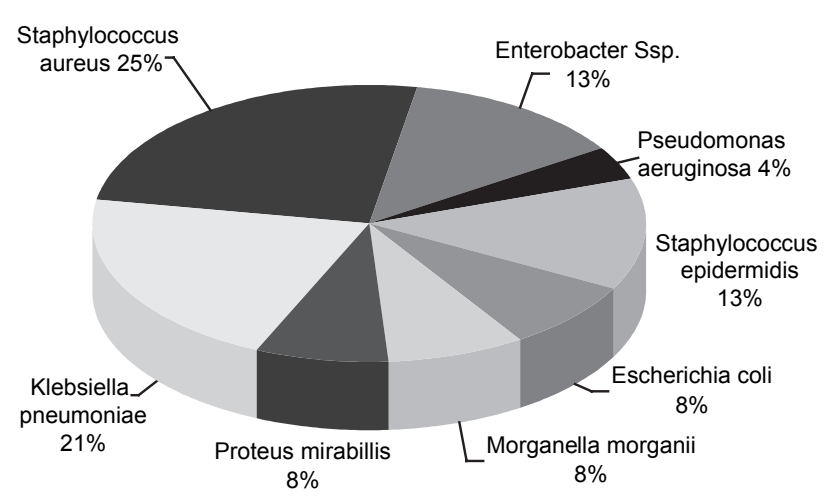

*Nota: O mesmo prontuário apresentava mais de um dos sinais e sintomas

Figura 4 - Patógenos encontrados nas culturas das feridas operatórias com mediastinite no Pronto Socorro Cardiológico da Universidade de Pernambuco - Recife, PE - 2007/2009 


\section{DISCUSSÃO}

A baixa prevalência encontrada no presente estudo está de acordo com as taxas semelhantes apontadas na literatura. No Brasil, observa-se em Minas Gerais uma prevalência de $2,1 \%$ da população estudada ${ }^{(5)}$.

O período transoperatório de cirurgia cardíaca é crítico para o paciente, devido a sua complexidade e procedimentos a ela inerentes, como a Circulação Extracorpórea (CEC) e o tempo intra-operatório prolongado. Vários fatores influenciam a incidência de infecção na ferida operatória, entre eles, os procedimentos invasivos e a defesa primária insuficiente provocada pelo trauma cirúrgico e pela Circulação Extracorpórea. Esta provoca alterações fisiológicas no sistema imunológico, especialmente pelo uso da hipotermia e hemodiluição, predispondo ao aparecimento de infecções, alterando a fisiologia normal do indivíduo ${ }^{(19)}$. Durante esse procedimento, alguns diagnósticos de enfermagem podem ser observados, como por exemplo: desequilíbrio no volume de líquidos, troca de gases prejudicada, alterações da proteção devido à inibição do sistema de coagulação com a heparinização sistêmica e seqüestro de leucócitos da circulação, inibindo a sua propriedade de fagocitar os germes invasores do organismo ${ }^{(2)}$. Com isso, cabe ao profissional de enfermagem estar preparado para intervir nessas situações.

Os fatores de co-morbidade mais encontrados também são associados às complicações cardiovasculares que necessitam de cuidados especiais e correção cirúrgica através da operação de revascularização do miocárdio. Com relação à mediastinite, esses fatores tornam-se sérios agravantes, pois, além de predispor a necessidade de intervenção cirúrgica, especialmente a hipertensão arterial sistêmica, influenciam de forma sistêmica no metabolismo do ser humano, como, por exemplo, o diabetes mellitus, o tabagismo e a obesidade que são dificultadores da cicatrização.

Nesse sentido, cabe à enfermagem a identificação precoce dessas condições ainda no período pré-operatório, a fim de planejar ações de controle dos mesmos através de práticas voltadas para educação em saúde e reduzindo danos previstos para o pós-operatório.

O uso de ponte mamária na operação de revascularização do miocárdio pode favorecer o desenvolvimento da mediastinite por causar hipóxia no esterno e tecidos locais facilitando a instalação e desenvolvimento de microorganismos infecciosos. Logo, o uso de duas mamárias aumenta potencialmente os riscos de infecção por diminuir o aporte sanguíneo para os músculos externais. Porém, o nosso estudo apresentou apenas o uso de uma mamária.

O elevado tempo de internação evidenciado no estudo corrobora com um maior risco de infecção, visto que aumenta as chances do paciente entrar em contato com agentes patógenos (contato com outros pacientes, com a equipe de saúde e com o ambiente hospitalar), além de contribuir para o aumento do estresse e da ansiedade e um quadro depressivo, podendo levar a diminuição de sua imunidade.

Os profissionais de saúde, em especial a enfermagem, devem estar atentos ao controle de infecções cruzadas durante os procedimentos invasivos. Ações simples como lavagem das mãos, uso adequado dos equipamentos de proteção individual (luvas, capotes e máscaras) e manutenção das técnicas assépticas, ajudam na prevenção da transmissão dos patógenos. Em hospitais-escola, tornam-se mais importante as medidas preventivas, visto o grande fluxo de pessoas (profissionais das diversas áreas e estudantes) nas unidades.

Os problemas levantados nessa pesquisa foram caracterizados com a finalidade de contribuir com as intervenções de enfermagem, para que seja obtida não só a solução do problema do paciente, mas para que seja fornecida a segurança necessária, fornecida pela fundamentação diagnóstica e favorecendo a tomada de decisão(22).

Foi observada a escassez do processo de enfermagem, bem como o preenchimento por esses profissionais dos prontuários (em enfermaria, UTI e CCIH), não permitindo o acompanhamento dos serviços prestados e como eles refletiram na evolução do quadro clínico dos pacientes.

\section{CONCLUSÃO}

A realização desse estudo demonstra a importância da assistência de enfermagem voltada ao atendimento de pacientes submetidos à cirurgia cardíaca, como uma relevante medida para prevenção e o controle do surgimento de novos casos de mediastinite, contribuindo para práticas de enfermagem segura e baixo índice de mortalidade para este agravo.

O enfermeiro assistencial, juntamente ao da Comissão de Controle de Infecção Hospitalar, é o profissional mais capacitado para atuar no controle da infecção hospitalar devido ao seu papel de maior proximidade e acompanhamento da evolução clínica do paciente, sendo o primeiro a identificar mudanças no seu quadro clínico.

Com base no estudo realizado, sugere-se a implantação da sistematização de assistência de enfermagem voltada ao paciente cirúrgico, incluindo a formulação e implementação de impressos para planejamento da assistência de enfermagem, que englobe os diagnósticos de enfermagem, metas e intervenções necessárias para vigilância epidemiológica; preenchimento completo do impresso próprio da Comissão de Controle de Infecção Hospitalar; descrição da localização, evolução e tratamento da ferida operatória; realizar evolução regular para acompanhar o progresso apresentado pelo doente; supervisão rotineira de enfermagem nos pacientes de pós-operatório imediato e tardio, para identificação precoce de sinais e sintomas; educação continuada e treinamento em serviço dos profissionais da equipe de enfermagem, favorecendo a resolutividade assistencial. 
1. Gelape CL. Infecção do sítio operatório em cirurgia cardíaca. Arq Bras Cardiol. 2007; 89(1):e3-9.

2. Almeida AR, Guedes MVC. Natureza, classificação e intervenções de enfermagem para pacientes com mediastinite. Rev Bras Enferm. 2008;61(4):470-5.

3. Guaragna JC, Facchi LM, Baiao CG, Cruz IBM, Bodanese LC, Albuquerque $L$, et al. Preditores de Mediastinite em Cirurgia Cardíaca. Rev Bras Cir Cardiovasc. 2004;19(2):165-70.

4. Mekontso-Dessap A, Kirsch M, Brun-Buisson C, Loisance D. Poststernotomy mediastinitis due to staphylococcus aureus: comparison of methicillin-resistant and methicillin-susceptible cases. Clin Infect Dis. 2001;32(6):877-83.

5. Sampaio DT, Alves JCR, Silva AF, Lobo Junior NC, Simões D, Faria W, et al. Mediastinite em cirurgia cardíaca: tratamento com Epíplon. Rev Bras Cir Cardiovasc. 2000;15(1):23-31.

6. Sancho LM, Minamoto H, Fernandez A, Sennes LU, Jatene FB. Descending necrotizing mediastinitis: a retrospective surgical experience. Eur J Cardiothorac Surg. 1999;16(2):200-5.

7. Salo JA, Isolauri JO, Heikkila LJ, Markkula HT, Heikkinen LO, Kivilaakso EO, et al. Management of delayed esophageal perforation with mediastinal sepsis. Esophagectomy or primary repair? J Thorac Cardiovasc Surg. 1993;106(6):1088-91.

8. Schimin LC, Batista RL, Mendonça FCC. Mediastinite no Hospital de Base do Distrito Federal: incidência em seis anos. Rev Bras Cir Cardiovasc. 2002;17(2):36-9.

9. Fatureto MC, Neves Júnior MA, Santana TC. Mediastinite aguda: análise retrospectiva de 21 casos. J Bras Pneumol. 2005;31(4):307-11.

10. Machado BL. Avaliação da resposta inflamatória em revascularização do miocárdio com circulação extracorpórea: correlação com a função pulmonar e evolução pós-operatória. [tese de doutorado]. São Paulo: Faculdade de Medicina, Universidade de São Paulo; 2004.

11. Abboud CS. Infecção em pós-operatório de cirurgia cardíaca. Rev Soc Cardiol Estado de São Paulo. 2001;5(5):915-21.

12. Magedanz EH. Elaboração de escore de risco para mediastinite em cirurgia de revascularização do miocárdio [tese doutorado]. Porto Alegre: Pontifícia Universidade Católica do Rio Grande do Sul; 2009.
13. Silva RRP. Prevalência de microorganismos isolados em mediastinite pós cirurgia cardíaca. Rev Med Minas Gerais. 1993;3(2):77-80.

14. Eagle KA, Guyton RA, Davidoff R, Ewy GA, Fonger J, Gardner TJ, et al. ACC/AHA Guidelines for Coronary Artery Bypass Graft Surgery: A Report of the American College of Cardiology/American Heart Association Task Force on Pratice Guidelines (Committee to Revise the 1991 Guidelines for Coronary Artery Bypass Graft Surgery). American College of Cardiology/American Heart Association. J Am Coll Cardiol. 1999;34(4):1262-347.

15. Braxton JH, Marrin CAS, McGrath PD, Ross CS, Morton JR, Norotsky $M$, et al. Mediastinitis and Long-Term Survival After Coronary Artery Bypass Graft Surgery. Ann Thorac Surg. 2000;70(6):2004-7.

16. Pereira MS, Souza ACS, Tipple AFV, Prado MA. A infecção hospitalar e suas implicações para o cuidar da enfermagem. Texto Contexto Enferm. 2005;14(2):250-7.

17. Cruz DALM, Pimenta CAM. Avaliação do doente com dor crônica em consulta de enfermagem: Proposta de instrumento segundo diagnóstico de enfermagem. Rev Latino Am Enferm. 1999;7(3):49-62.

18. Universidade de Pernambuco; Pronto Socorro Cardiológico de Pernambuco; Comissão de Controle de Infecções Hospitalares. Censo de infecções hospitalares. Recife; 2009.

19. Campos YAR. A circulação extracorpórea como fator predisponente da infecção hospitalar. Perfusion Line [Internet]. 2006 [citado 2010 maio 13]. Disponível em: http://perfline. com/artigos/ih_e_cec.pdf

20. Galdeano LE, Rossi LA, Nobre LF, Ignácio DS. Diagnósticos de enfermagem de pacientes no período transoperatório de cirurgia cardíaca. Rev Latino Am Enferm. 2003;11(2):199-206.

21. Guedes GP, Barbosa YRA, Holanda G. Correlação entre força muscular respiratória e tempo de internação pós-operatório. Fisioter Mov. 2009;22(4):605-14.

22. Nascimento KC, Backes DS, Koerich MS, Erdmann AL. Sistematização da Assistência de Enfermagem: vislumbrando um cuidado interativo, complementar e multiprofissional. Rev Esc Enferm USP. 2008;42(2):643-8. 